\title{
Contribution of HLA Class II Genes, DRB4*01:01, DRB1*07:01, and DQB1*03:03:2 to Clinical Features of Vitiligo Disease in Iranian Population
}

\author{
Roya Ghaffarnia \\ Tehran University of Medical Sciences \\ Zahra Saffarian \\ Emam Khomeini Hospital \\ Majid Shahbazi \\ Golestan University of Medical Sciences and Health Services \\ Mahdi Zamani (D Mzamani@tums.ac.ir) \\ Tehran University of Medical Sciences https://orcid.org/0000-0002-3524-5425
}

\section{Research Article}

Keywords: Vitiligo, HLA-DRB1, HLA-DRB4, HLA-DQB1, PCR-SSP

Posted Date: August 19th, 2021

DOI: https://doi.org/10.21203/rs.3.rs-813285/v1

License: @ (1) This work is licensed under a Creative Commons Attribution 4.0 International License. Read Full License

Version of Record: A version of this preprint was published at Molecular Biology Reports on October 22nd, 2021. See the published version at https://doi.org/10.1007/s11033-021-06855-3. 


\section{Abstract}

Background: Vitiligo is a multifactorial depigmentation condition, which is due to skin melanocyte destruction. The increased expression of HLA class II genes in patients with pre-lesions of Vitiligo suggests an important role for the participation of immune response in the Vitiligo development. Recent studies progressively focused on HLA-DRB1 and DQB1 genes. In this study, we have evaluated the association and role of HLA-DRB4*01:01, -DRB1*07:01, and -DQB1*03:03:2 genes in different clinical subtypes of Vitiligo in the Iranian population.

Methods: First, Genomic DNA from peripheral blood of 125 unrelated Vitiligo patients and 100 unrelated healthy controls were extracted through salting-out method. Then, HLA CLASS II genotyping were performed using sequence-specific primer PCR method. Finally, the clinical relevance of the testing for these genotypes were evaluated by applying the PcPPV (prevalence-corrected positive predictive value) formula.

Results: Our results indicated the positive associations of DRB4*01:01 and DRB1*07:01 allelic genes with early-onset Vitiligo $(P=$ 0.024 and 0.022 , respectively). The $D R B 4 * 01: 01$ also showed a strong protection against late-onset Vitiligo $(P=0.0016, R R=0.360)$. Moreover, our data revealed that the DRB1*07:01 increases the susceptibility to Sporadic Vitiligo $(P=0.030, R R=1.702)$. Furthermore, our findings proposed that elevated vulnerability of Vitiligo patients due to DRB4*01:01 and DRB1*07:01 alleles may be is correlated with the presence of amino acid Arginine at position 71 at pocket 4 on the antigen-binding site of the HLA-DRB1 receptor.

Conclusion: Our findings on different subtypes of Vitiligo suggest that, despite a more apparent autoimmune involvement, a nonautoimmune nature for the etiology of Vitiligo could also be considered.

\section{Introduction}

Vitiligo is a multifactorial systemic depigmentation condition, characterized by non-scaly macules, with distinct sharp margins, which is partly due to melanocyte destruction in the affected area of the skin. The prevalence of this skin disorder is estimated to be about $1 \%[1]$. The autoimmune origin with an inherent genetic background leading to melanocyte destruction has been emphasized to be a remarkable endogenous factor in Vitiligo pathogenesis[2]. The various phenotypes, and different patterns of Vitiligo inheritance might reflect the fact that different subtypes of Vitiligo have different principal genetic architectures[3]. The discrepancies in genes associated with different subtypes of Vitiligo also demonstrate the different pathology of Vitiligo subtypes, and heterogeneity in the genetic background of clinical features of Vitiligo.

Several genome-wide association studies (GWAS) have been conducted, and more than 30 genes have been reported as predisposing factors for Vitiligo [4]. Specifically, given the frequent co-occurrence of Vitiligo with autoimmune diseases, there have been investigations of possible HLA associations in Vitiligo. Despite the variable results of case-control association studies, HLA alleles have been positively associated with Vitiligo in several populations. According to recent review articles, particular HLA alleles such as HLA-DRB1*07:01,-DQB1*03:03:2, and-DRB4*01:01 have been frequently found to be positively associated with Vitiligo, and considered to serve an important role in Vitiligo predisposition[5, 6].

It is believed that the association of HLA alleles with a disease can be explained by variations in amino acid signatures of peptidebinding pockets of HLA molecules[7].The major binding pockets of HLA Class II are P1, P4, P6, P9, which can possibly affect the peptide binding selectivity[8]. Previous studies declared that sequence modifications in these binding regions,can markedly influence the T cell specificity, and proper immune response[9]. Singh et al. also reported considerable differences in the amino acid signature of the peptide-binding pockets for the HLA-DR 3 chain in Vitiligo patients and healthy individuals[10].

Previous studies in Iranian population have been indicated the association of CD4 [11], and MTHFR C677T genes with Vitiligo disease [12], while the association of HLA alleles with Vitiligo are not investigated in Iranian population so far. Hence, it was interesting to study the possible contribution of frequently associated HLA alleles with Vitiligo disease. In the present study, we evaluate the possible association of HLA-DRB1*07:01, -DRB4*01:01, and -DQB1*03:03:2 with Vitiligo and also, with different clinical features of Vitiligo in Iranian population. In addition, we investigate the influence of amino acid residue of HLA-DRB1*07:01, and DR $34{ }^{\star}$ 01:01 chains on susceptibility to Vitiligo in Iranian patients.

\section{Materials And Methods}




\subsection{Patients and Controls}

One hundred twenty-five unrelated Vitiligo patients ( 56 males and 69 females, median age of $\simeq 44$ years) were recruited from the Department of Dermatology, Imam Khomeini Hospital Complex at Tehran. The patients were compared with 100 unrelated healthy controls who were fully matched by age, gender, and ethnicity. All the patients and control subjects were of Iranian origin. The diagnosis is confirmed by experienced dermatologists, based on clinical symptoms after a complete clinical examination, skin biopsy, and medical histories like the presence of generalized, non-segmental white macules (Köbner's phenomenon), and activity or stability of the disease.

We obtained consent and questionnaire forms for each patient and control individuals before their participation in the study. The family history, age of onset, psychological trauma, clinical classification, and response to treatment were obtained from each patient. Patients who took immunosuppressive drugs were excluded from the study because of the response to treatment screening. The control group consisted of 100 age, gender, and ethnic background matched healthy individuals, with no family history of Vitiligo or autoimmune diseases. The study was approved by the ethics committee and review board of Tehran University of Medical Sciences.

\subsection{HLA Genotyping and Polymorphic Amino Acids Analysis}

Genomic DNA from the peripheral blood of the healthy controls and patients was extracted by the salting-out method. HLA Genotyping was performed using PCR sequence specific primer (PCR-SSP) method. This study provided a high resolution determination of HLA class II genes using primers presented in Table 1[13]. PCR cycling parameters employed are as follows: DNA denaturation for $1 \mathrm{~min}$ at $94^{\circ} \mathrm{C}$ followed by five cycles of polymerization each $25 \mathrm{Sec}$ at $94^{\circ} \mathrm{C}, 45 \mathrm{Sec}$ at $70^{\circ} \mathrm{C}$, and $45 \mathrm{Sec}$ at $72^{\circ} \mathrm{C}$, then 21 cycles of polymerization each $30 \mathrm{Sec}$ at $94^{\circ} \mathrm{C}, 50 \mathrm{Sec}$ at $65^{\circ} \mathrm{C}, 45 \mathrm{Sec}$ at $72^{\circ} \mathrm{C}$, and as final extension reaction four cycles each $25 \mathrm{Sec}$ at $94^{\circ} \mathrm{C}, 1 \mathrm{~min}$ at $55^{\circ} \mathrm{C}, 3 \mathrm{~min}$ at $72^{\circ} \mathrm{C}$ and $1 \mathrm{~min}$ at $72^{\circ} \mathrm{C}[13]$.

Table 1

Primers sequence used in the study

\begin{tabular}{|lll|}
\hline Primer & Primer sequence $\mathbf{5}^{\prime} \boldsymbol{\rightarrow} \mathbf{3}^{\prime}$ & Molecular weight $(\mathbf{b p})$ \\
\hline DRB4*01:01 & GATCGTTCGTGTCCCCACAA & 151 \\
& TCCCCCAGGTCACTGTTGT & \\
\hline DRB1*07:01 & $\begin{array}{l}\text { CCCGTAGTTGTGTCTGCACAC } \\
\text { CCTGTGGCAGGGTAAGTATA }\end{array}$ & 232 \\
& GACGGAGCGCGTGCGTTA & 129 \\
& CTGTTCCAGTACTCGGCGT & \\
\hline
\end{tabular}

PCR products were separated on $6 \%$ polyacrylamide gels. DNA fragment bands were set against 50 bp DNA ladder, after electrophoresis. Each band compared with the ladder to identify the approximate size of fragments matching to the particular alleles. We used Ethidium bromide (EtBr) staining to detect the presence of the bands.

All the polymorphic sites of HLA-DR $11^{\star} 0701$, and $-D R \beta 4 * 01: 01$ chains which are located in the functionally important position of the DRß1 chain were analyzed. The analysis was based on the three-dimensional structure of the HLA-DR 3 chain [14]. All of the amino acids were determined from the nucleotide sequences of alleles. In this study, amino acid position 71 located in the functionally important site of the antigen-binding groove of the DRß1 chain, which can encode Lys, Arg, Ala, or Glu amino acids was investigated. To identify the specific amino acids in different alleles, the HLA Epitope Registry database was used (https://www.epregistry.com.br/).

\subsection{Statistical Analysis}

The significance of the distribution of the allelic and phenotype frequencies between patients and healthy control was compared by the Fisher exact test as appropriate. The data, including genotyping of DRB4*01:01/X, DRB1*07:01/X, and DQB1*03:03:2/X, inserted into a database, employing SPSS for Windows version 25 (Chicago, IL, USA). Fisher exact tests were employed for statistical 
analysis of data. $\mathrm{P}<0.05$ was considered as significant. Odds ratios (OR) or Relative Risk (RR) and their $95 \%$ confidence intervals $(\mathrm{Cl})$ were also calculated. Moreover, the predictive values of the test were calculated by prevalence corrected positive

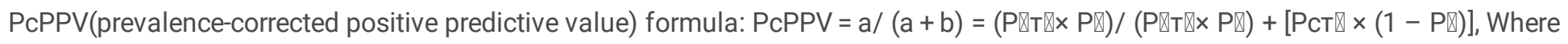

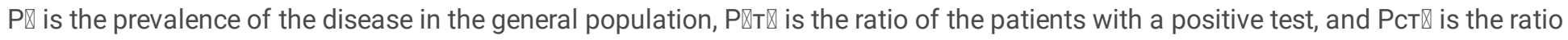
of the controls with a positive test [15].

The Correlation between response to treatments and different genotypes were computed using $\triangle \mathrm{BR}$ and $\triangle \mathrm{GR}$ formula, where $\Delta \mathrm{BR}=$ Bad response of genotype - Bad response of total, and $\Delta \mathrm{GR}=\mathrm{Good}$ response of genotype - Good response of total.

\section{Results}

Of the 125 patients included in this study, 49 people had a positive family history, and 76 people had sporadic Vitiligo. In this study, the association of HLA-DRB4*01:01, DRB1*07:01, and DQB1*03:03:2 genes with different clinical subtypes of Vitiligo were assessed in the Iranian population (Table 2). Furthermore, the possible correlation between these alleles with response to Vitiligo treatment was investigated (Table 3) 
Table 2

Frequency of HLA genotypes in different types of Vitiligo Disease compared with Control

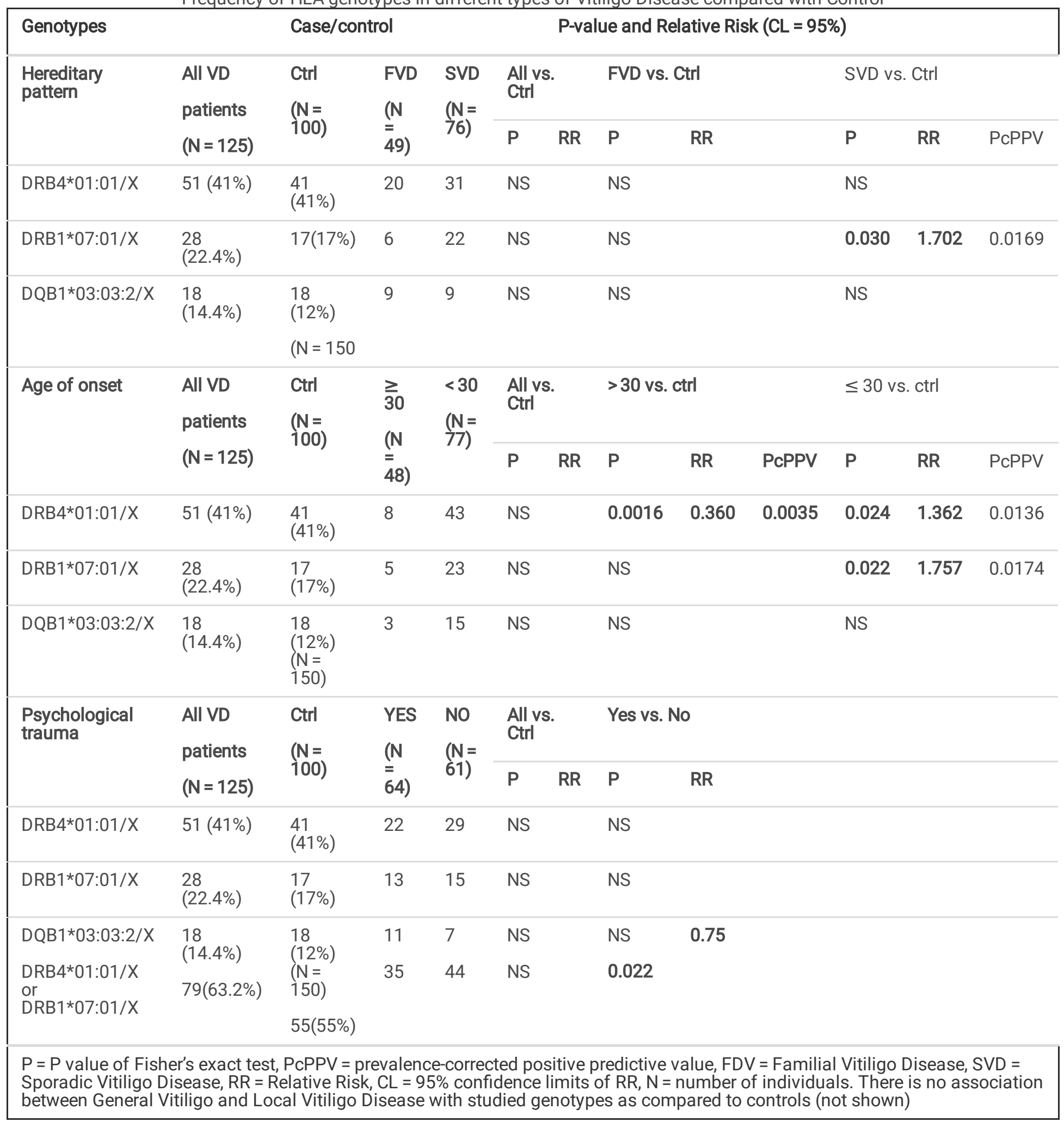


Table 3

Response to common treatments for Vitiligo patients with different HLA genotype

\begin{tabular}{|c|c|c|c|c|}
\hline Genotypes & Bad response & Good response & $\Delta \mathrm{BR}$ & $\Delta \mathrm{GR}$ \\
\hline HLA-DRB4*01:01/X & $35 / 51(68.7 \%)$ & 16/51 (31.3\%) & +5.4 & \\
\hline HLA-DRB1*07:01/X & $17 / 28(60.8 \%)$ & $11 / 28(39.2 \%)$ & & +3.1 \\
\hline HLA-DQB1*03:03:2/X & $11 / 18(61.2 \%)$ & $7 / 18(38.8 \%)$ & & +2.7 \\
\hline Total & $45 / 71(63.3 \%)$ & $26 / 71(36.1 \%)$ & - & - \\
\hline
\end{tabular}

\subsection{Association of HLA genotypes with Familial (FVD) and Sporadic (SVD) Vitiligo}

After stratification of familial or Sporadic Vitiligo patients, the result of statistical analysis indicated a significant association of HLA-DRB1*07:01/X genotype with the Sporadic Vitiligo patients (Table 2; P-value $=0.030, R R=1.702)$. Further analysis did not show any significant association between other genotypes and familial or Sporadic Vitiligo as compared with control (Table 2).

\subsection{Association of HLA genotypes with early or late-onset Vitiligo}

After stratification of early and late-onset Vitiligo patients, the statistical analysis demonstrated that HLA-DRB4*01:01/X genotypes imposes a significant susceptibility for early-onset Vitiligo when compared to control individuals (Table 2; P-value $=0.024, R R=$ 1.362 , PcPPV $=0.0136$ ). The results also showed that the frequency of HLA-DRB4*01:01/X genotype is significantly decreased in patients with age $>30$ as compared to controls and indicating strong protection against late-onset Vitiligo development (Table 2; Pvalue $=0.0016, \mathrm{RR}=0.360, \mathrm{PcPPV}=0.0035$ ). Moreover, Genotype analysis revealed that the frequency of HLA-DRB1*07:01/X genotype is statistically increased in patients with early-onset Vitiligo $(\leq 30)$ when compared to controls. This genotype shows a significant positive association with early-onset of Vitiligo (Table 2; P-value $=0.022, R R=1.757$, PcPPV $=0.0174$ ).

\subsection{Association of genotypes with psychological trauma}

We evaluated the possible association of HLA alleles with Vitiligo disease in patients with psychological trauma and nonpsychological trauma, which is examined for the first time. When Vitiligo patients with psychological trauma were compared to Vitiligo patients without psychological trauma, the patients carrying DRB4*01:01/X or DRB1*07:01/X genotypes (frequencies of carriers and non-carriers are 0.54 and 0.72 respectively) showed a negative association with psychological trauma (Table 2; P-value $=0.022, \mathrm{RR}=0.75$ ).

\subsection{Correlation between response to treatments for Vitiligo and different genotypes}

In the context of genotypes studied here, patients were analyzed for their therapeutic response to common medications for Vitiligo, including Light therapy, ELIDEL $\circledast$ (pimecrolimus), OXSORALEN-ULTRA® capsules (methoxsalen), and Ganoderma lucidum mushrooms. The obtained data indicated that patients carrying the DRB4*01:01/X genotype exhibited the worst response for the Vitiligo treatment $(\triangle B R=+5.4)$. While patients having HLA-DRB1*07:01/X and HLA-DQB1*03:03:2/X exhibited positive response to Vitiligo drug therapy ( $\triangle \mathrm{GR}=3.1$, and $\triangle \mathrm{GR}=2.7$ respectively). The response to common Vitiligo treatments in the context of the selected HLA genotypes is shown in Table 3.

\subsection{Clinical significance of the HLA-DRB4*01:01/X, HLA-DRB1*07:01/X and HLA-DQB1*03:03:2/X genotypes}

Relative risk is a ratio of probabilities. It compares the occurrence or risk (incidence proportion) of an outcome between those with a particular exposure versus a non-exposed group. Thus, we calculated the Prevalence corrected Positive Predictive Values (PcPPV) of the test to show the influence of associated genes in the susceptibility to Vitiligo and to evaluate the absolute risk of disease caused by these genes [15]. In other words, we defined and determined meaningful PcPPV which could have diagnostic relevance for the health practitioners. 
The PcPPV of testing for HLA-DRB4*01:01/X and HLA-DRB1*07:01/X genotypes were calculated for subtypes of Vitiligo (Table 2). As compared with the prevalence of Vitiligo in Caucasians $(0.5 \pm 1 \%)$, and assuming the prevalence of Vitiligo in Iranian population to be $1 \%$, the PCPPV of testing for positive association of DRB1*07:01/X genotype with SVD and early-onset of Vitiligo, were $1.69 \%$ and $1.74 \%$ respectively (Table 2 ).In other words, individuals who are carrying the DRB1*07:01/X genotype have $1.69 \%$ absolute risk to develop SVD and 1.36\% absolute risk of developing early onset Vitiligo. The PcPPV of testing for the DRB4*01:01/X genotype, which provides a strong protection against late-onset of Vitiligo, was $0.35 \%$, significantly lower than the prevalence of Vitiligo in the general population (PcPPV $=1 \%)$.

\section{Discussion}

We studied the associations of HLA-DRB4*01:01, DRB1*07:01, and DQB1*03:03:2 genes and their amino acids located in functionally important positions of their protein chains with Vitiligo disorder. The co-occurrence of Vitiligo with other autoimmune disease reveals a common origin and genetic predisposition inducing autoimmunity, as the expression of HLA class II molecules is increased in the pre-lesional epidermis, the sites of melanocyte destruction and skin depigmentation in Vitiligo[16].The specific increase in the levels of HLA class II but not the class I molecules in the pre-lesions of Vitiligo patients suggests the prominent role of the HLA class II gene products in predisposition to Vitiligo skin disease. It is considered that a right level expression of the HLA antigen-presenting receptors and a strong interaction between HLA antigen-presenting receptors with T cell receptors is required for the proper immune response Overexpression of HLA class II molecules may modify the T-cell receptor assembly due to T-cell maturation in the thymus and consequently, influence the survival and development of mature CD $4+T$ cells. The converted CD $4+T$ cells have cytotoxic activity (CD4 CTL), and in terms of Vitiligo disorder, destruction of melanocytes[17].

Over $30 \%$ of the entire genetic effect for Vitiligo is suggested to be due to abnormal expression of different HLA genes[18], particularly, the alleles of DRB and DQB genes. In the present study, after stratifying these patients, based on family history, age of onset, and psychological trauma before the disease onset, the association of DRB4, DRB1, and DQB1 allelic genes with these distinct subtypes of Vitiligo were revealed.

Recent studies indicated differences in HLA frequencies between FVD (Familial Vitiligo Disease) and SVD (Sporadic Vitiligo Disease) patients which suggests a different genetic background and etiology for familial and non-familial Vitiligo [19]. When we divided the patients in FVD and SVD groups, our results showed a significant increase in the frequency of DRB1*07:01/X genotype in SVD patients $(P=0.030)$. This finding is in agreement with studies showing the association of DRB1*07:01/X genotype with susceptibility to SVD[20]. There is two hypotheses which could explain the different etiology for these two forms: 1 . FVD could be the result of an immune attack against melanocytes presenting HLA Class II alleles; 2. SVD may be due to viral infections that could induce the expression of HLA II genes[21]. In addition, it has been shown that sporadic Vitiligo patients are at greater risk for certain autoimmune/auto inflammatory diseases [22]. Our finding is in line with the latter pathogenesis of Vitiligo.

We further stratified our patient groups by age to early-onset (under 30) and late-onset (above 30) divisions. Divided samples revealed that frequencies of HLA-DRB4*01:01 and DRB1*07:01 genes to be highly increased in early-onset patients as compared with healthy individuals $(P=0.024, P=0.030$ respectively). Our observations are in line with the study of Xiaojing et al., which reported an association of $\mathrm{DRB} 1 * 07: 01 / \mathrm{X}$ genotype with the earlier onset of the disease in the Uygar population [23].Our results also indicate a strong negative association of HLA-DRB4*01:01 with the late-onset Vitiligo $(P=0.0016, R R=0.362)$, which confirms several studies involving Dutch patients [24]. The above data taken together, suggest susceptibility of HLA-DRB4*01:01 to earlyonset of the disease $(P=0.024, R R=1.362)$, with an autoimmune basis, and a more significant protective role for HLA-DRB4*01:01 to late-onset Vitiligo $(P=0.0016, R R=0.360)$, with a non-immune etiology.

To extend the present study, we analyzed the correlation between DRB4*01:01, DRB1*07:01, and DQB1*03:03:2 and psychological trauma before Vitiligo infection in the Iranian population, an association analysis for Vitiligo that has not been addressed before. Our results showed that patients with a psychological trauma before infection who are carrying DRB4*01:01/X or DRB1*07:01/X genotypes have a decreased risk for Vitiligo development $(P=0.022, R R=0.75)$ whereas, the frequency of $D R B 4 * 01: 01 / X$ or DRB1*07:01/X genotypes are increased in patients without psychological Trauma. These findings may suggest that the development of Vitiligo in patients with psychological trauma before infection may be caused by non-autoimmune event/s in contrast with Vitiligo patients without psychological trauma. 
The relative risk or odd ratio, used in case-control studies provides the probability of risk alone. Thus, we calculated PcPPV to estimate the absolute risk and effectiveness of testing for selected genes [15]. For instance, based on the assumption that the prevalence of SVD in the Iranian patients is $1 \%$, PCPPV of DRB $* 07: 01 / \mathrm{X}$ genotype for SVD in our patient group is about 1.69 . This indicates that patients carrying the DRB1*07:01/X genotype provide 1.69\% absolute risk for developing SVD. In other words, in comparison with the prevalence of SVD $(1 \%)$ in the general Iranian population, the risk of individuals carrying the DRB1*07:01/X genotype to develop SVD is increased by $0.69 \%$.

Several studies reported that special amino acid residues in the HLA class II structure, especially in the pockets of antigen-binding grooves could determine the affinity of HLA molecules. These amino acid signatures are supposed to be critical for enhanced disease susceptibility. Both HLA-DRB4*01:01 and DRB1*07:01 molecules, shown in this study to be associated with Vitiligo, have the positively charged amino acid Arginine at position 71, situated within the pocket 4 (P4) of their antigen-binding groove. Arg71 $\beta$ is also shown to be associate with other autoimmune disorders [25]. Our findings proposed that elevated vulnerability of Vitiligo patients due to DRB4*01:01 and DRB1*07:01 alleles may be is correlated with the presence of amino acid Arginine at position 71 at pocket 4 on the antigen-binding site of the HLA-DRB1 receptor.

It is important to note that the pathogenesis of Vitiligo is complex and that the autoimmune nature of this disease is still under investigation. Nevertheless, despite the possible involvement of the non-autoimmune factors, including abnormal melanocytes or environmental factors that might complicate the understanding of the disease, due to accumulating data implicating different components of the immune system, the etiology for Vitiligo disorder is considered to be more of an autoimmune nature. On the other hand, several subtypes of Vitiligo do not show association with the HLA alleles as crucial elements of the autoimmune system, which might define a non-autoimmune etiology for the development of the disease. This contradiction is also observed in our Vitiligo patient group demonstrating a mixture of autoimmune and non-autoimmune genetic associations.

In conclusion, HLA-DRB1*07:01 allele is positively associated with sporadic form of Vitiligo and, HLA-DRB4*01:01, and DRB1*07:01 alleles are associate with the susceptibility to early-onset Vitiligo. Moreover, HLA-DRB4*01:01 allele provides protection for late-onset Vitiligo and our results also suggest for the first time that Vitiligo disorder in patients with psychological trauma before infection may has a non-autoimmune etiology.

\section{Declarations}

\section{Acknowledgements:}

We appreciate all members of the Department of Dermatology, Imam Khomeini Hospital Complex for their assistance in collecting samples used in this study, as well as the patients and their families, whose help and participation made this work possible. This study was certified by the ethics committee and review board of the Tehran University of Medical Sciences.

\section{Funding sources statement:}

This study was certified by the ethics committee and review board of the Tehran University of Medical Sciences.

\section{Conflict of interests:}

The authors have no conflicts of interest to declare that are relevant to the content of this article.

\section{References}

1. Rahman R, Hasija YJBD, Exploring vitiligo susceptibility and management: a brief review. (2018) 2(1): p. 1-13

2. Wankowicz-Kalinska A et al (2004) Autoimmune aspects of depigmentation in vitiligo. in Journal of Investigative Dermatology Symposium Proceedings. Elsevier

3. Roberts GHL (2019) Genetic architecture of vitiligo and vitiligo subtypes, The. University of Colorado at Denver, Anschutz Medical Campus 
4. Ghosh SJIJoD, Chemical vitiligo: A subset of vitiligo. (2020) 65(6): p. 443

5. Said-Fernandez SL et al., Novel immunological and genetic factors associated with vitiligo: A review. 2021. 21(4): p. 1-1

6. Zhang X-J, Chen J-J (2005) and J.-B.J.J.o.d.s. Liu. The genetic concept of vitiligo 39(3):137-146

7. Shin D-H et al., HLA alleles, especially amino-acid signatures of HLA-DPB1, might contribute to the molecular pathogenesis of early-onset autoimmune thyroid disease. 2019. 14(5): p. e0216941

8. Rappazzo CG, Huisman BD, and M.E.J.N.c. Birnbaum, Repertoire-scale determination of class II MHC peptide binding via yeast display improves antigen prediction. 2020. 11(1): p. 1-14

9. Cole DK et al., Modification of MHC anchor residues generates heteroclitic peptides that alter TCR binding and T cell recognition. 2010. 185(4): p. 2600-2610

10. Singh A et al., HLA alleles and amino-acid signatures of the peptide-binding pockets of HLA molecules in vitiligo. 2012. 132(1): p. $124-134$

11. Zamani et al., Possible association of the CD4 gene polymorphism with vitiligo in an Iranian population. 2010. 35(5): p. 521524

12. Bagheri Hamidi A et al., Association of MTHFR C677T polymorphism with elevated homocysteine level and disease development in vitiligo. 2020. 47(4): p. 342-350

13. Bunce $M$ et al., Phototyping: comprehensive DNA typing for HLA-A, B, C, DRB1, DRB3, DRB4, DRB5 \& DQB1 by PCR with 144 primer mixes utilizing sequence-specific primers (PCR-SSP). 1995. 46(5): p. 355-367

14. e Oliveira DMT et al., pHLA3D: Updating the database of predicted three-dimensional structures of HLA with HLA-DR, HLA-DQ and HLA-DP molecules. 2021. 82(1): p. 8-10

15. Zamani M (1998) and J.J.J.A.j.o.m.g. Cassiman, Reevaluation of the importance of polymorphic HLA class I/ alleles and amino acids in the susceptibility of individuals of different populations to type I diabetes. Am J Med Genet 76(2):183-194

16. Le Poole I et al (2003) Expansion of vitiligo lesions is associated with reduced epidermal CDw60 expression and increased expression of HLA-DR in perilesional skin. Br J Dermatol 149(4):739-748

17. Takeuchi A and T.J.F.i.i. Saito, CD4 CTL, a cytotoxic subset of CD4 + T cells, their differentiation and function. 2017. 8: p. 194

18. Fain PR et al., HLA class II haplotype DRB1*04-DQB1*0301 contributes to risk of familial generalized vitiligo and early disease onset. 2006. 19(1): p. 51-57

19. Deo KS, S.A.J.I.J.o R, Ganjre (2018) Genetics of multiplex familial vitiligo as cases and controls: a preliminary report. Int J Res 4(3):403

20. Abdullah GA et al., The association between Human Leukocyte Antigen-DRB1 and vitiligo. 2015(4): p. 190-194

21. Stevanović $S$ et al., HLA class II upregulation during viral infection leads to HLA-DP-directed graft-versus-host disease after CD4 + donor lymphocyte infusion. 2013. 122(11): p. 1963-1973

22. Alkhateeb A et al (2003) Epidemiology of vitiligo and associated autoimmune diseases in Caucasian probands and their families. Pigment Cell Res 16(3):208-214

23. Kang $X$ et al (2017) Association of HLA-DRB1* 1201/02, DRB1* 0701/02, DQA1* 0302 and DQB1* 0303 Alleles with population Uygur patients of vitiligo. Clon Transgen 6(157):2

24. Zamani et al., Linkage and association of HLA class II genes with vitiligo in a Dutch population. 2001. 145(1): p. 90-94

25. Ling SF et al., HLA-DRB1 amino acid positions $11 / 13,71$, and 74 are associated with inflammation level, disease activity, and the health assessment questionnaire score in patients with inflammatory polyarthritis. 2016. 68(11): p. 2618-2628 